\title{
Clinico-radiological and Functional outcome of Unstable Intertrochanteric Fractures by Proximal Femoral Nail Antirotation-2 (PFN-A2)
}

ISSN: 2576-8875

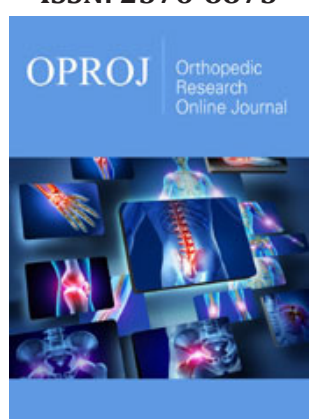

${ }^{* 1}$ Corresponding author: Druva V, Department of Orthopaedics, SS Institute of Medical Sciences and Research Center, Davangere, Karnataka, India

Submission:

Published: 侮 October 22, 2019

Volume 6 - Issue 1

How to cite this article: Manjunath J, Venkataramana Rao M, Srinath SR, Kiran GU, Shashidhara H, et al. Clinicoradiological and Functional outcome of Unstable Intertrochanteric Fractures by Proximal Femoral Nail Antirotation-2 (PFN-A2). Ortho Res Online J. 6(1). OPROJ.000630.2019.

DOI: $10.31031 /$ OPROJ.2019.06.000630

Copyright@: Druva V, This article is distributed under the terms of the Creative Commons Attribution 4.0 International License, which permits unrestricted use and redistribution provided that the original author and source are credited.

\author{
Manjunath J, Venkataramana Rao M, Srinath SR, Kiran GU, Shashidhara H and \\ Druva $V^{*}$ \\ Department of Orthopaedics, India
}

\begin{abstract}
Background: Unstable intertrochanteric fractures were one of the most common fractures of the hip especially in the elderly with osteoporotic bones, usually due to low-energy trauma and in young adults due to high velocity trauma. The incidence of these fractures was rising because of increasing number of senior citizens with osteoporosis. The problems associated with unstable intertrochanteric fractures were due to substantial morbidity and mortality, malunion, implant failure and associated medical conditions. Therefore, this has led to the development of intramedullary devices in the management of unstable intertrochanteric fractures.
\end{abstract}

Objective: To analyze clinico-radiological and functional outcome of unstable intertrochanteric fractures by proximal femoral nail antirotation A2 (PFN-A2).

Materials and methods: A prospective study with 73 adult patients with unstable intertrochanteric fractures according to Boyd \& Griffin classification were treated with proximal femoral nail antirotation-2 (PFN-A2) in SSIMS, Davangere from October 2017 and September 2019. All the cases were followed at regular intervals as per our study protocol. The functional outcome were assessed with Harris Hip Score.

Results: In the present study, 73 unstable intertrochanteric fractures underwent surgical fixation with proximal femoral nail antirotation 2 (PFN-A2). The functional results assessed by Harris Hip score showed excellent in 41 cases (56.16\%), good in 15 cases $(20.54 \%)$, fair in 12 cases $(16.43 \%)$ and poor in 5 cases $(6.84 \%)$.

Conclusion: We conclude that the proximal femoral nail antirotation 2 (PFN-A2) was an ideal implant for unstable intertrochanteric fractures, leading to high rate of bone union restoring the lateral femoral wall, reducing the chances of implant failure and decreasing the post-operative morbidity by improving the functional quality of life.

Keywords: Unstable intertrochanteric fractures; PFN-A2; Boyd \& Griffin; Cephalomedullary

\section{Introduction}

Intertrochanteric fracture is the fracture of the proximal femur, in which the fracture line extends from the greater trochanter to lesser trochanter. These fractures are common in patients in geriatric age group, due to increase in prevalence of osteoporosis. However, it may also occur in young adults due to high velocity trauma. Nine out of ten intertrochanteric fractures occur in elderly, the female to male ratio is 3:1 [1].

These fractures are usually classified as Stable and Unstable Fractures. The characteristics of unstable variety are posteromedial fragmentation, basicervical patterns, reverse obliquity patterns, displaced greater trochanteric (lateral wall communition) fractures [2]. These fractures can be treated both conservatively and operatively, due to higher risk of mortality and morbidity associated with conservative management. There is a need for internal fixation for early mobilization to avoid complications [3].

The focus of surgical research regarding internal fixation in late $20^{\text {th }}$ century was to minimize implant failure and cut out of the femoral head and neck fixation components, with the complicit acceptance of loss of reduction of the fracture [4]. Rigid internal fixation and early mobilization is the present standard method of treatment [5]. The current practice of treatment of stable variety of intertrochanteric fracture with dynamic hip screw is widely 
accepted, however unstable variety of intertrochanteric fractures are better treated with cephalomedullary nails [6].

Cephalomedullary device has many potential advantages like more efficient load transfer, provides resistance to varus collapse and provides better rotational stability of the head, the amount of sliding is limited by intramedullary location, therefore less chance of shortening and deformity with shorter operative time, less soft tissue dissection and less blood loss [7-9]. This study was done to analyze clinico-radiological and functional outcome of surgical management of unstable intertrochanteric fractures by PFN-A2.

\section{Materials and Methods}

With a level IV evidence, a prospective study was carried out from October 2017 and September 2019 in department of Orthopaedics, SS Institute of Medical Sciences \& Research Centre, Davanagere, Karnataka. A total of 73 patients with unstable intertrochanteric fractures were treated surgically by proximal femoral nail - A2 and were followed up at the immediate post op period and at the end of 1, 2,6, 12 and 18 months and evaluated for
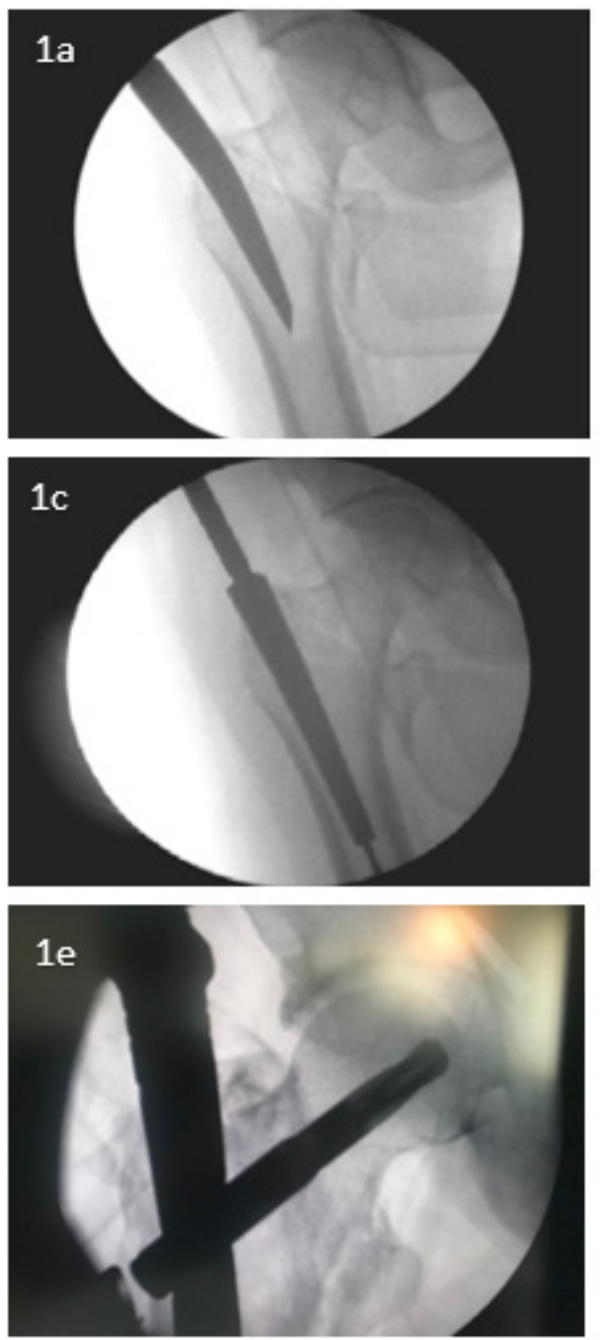

clinic-functional outcome using Modified Harris Hip score.

Patients who were in the age group from 30-80 years of age, patients with clinically and radiologically diagnosed unstable intertrochanteric fractures as per Body and Griffin classification were included in the study. Patients who were below 30 and above 80 years of age, patients with clinically and radiologically diagnosed stable intertrochanteric fractures as per Body and Griffin classification patients with compound fractures, pathological fractures and fractures over ipsilateral lower limb and patients with neck of femur and shaft of femur fractures were excluded from the study.

After getting IEC and informed \& written consent from the patients and attenders, the patients enrolled in the study were subjected for thorough clinical examination. The baseline investigations and radiographic analysis such as plain X ray of the affected hip with proximal femur were analysed. The degree of osteoporosis was evaluated by Singh's index. All the cases were posted for surgical management with closed or open reduction with PFN-A2 and followed up according to our study protocol (Figure 1).
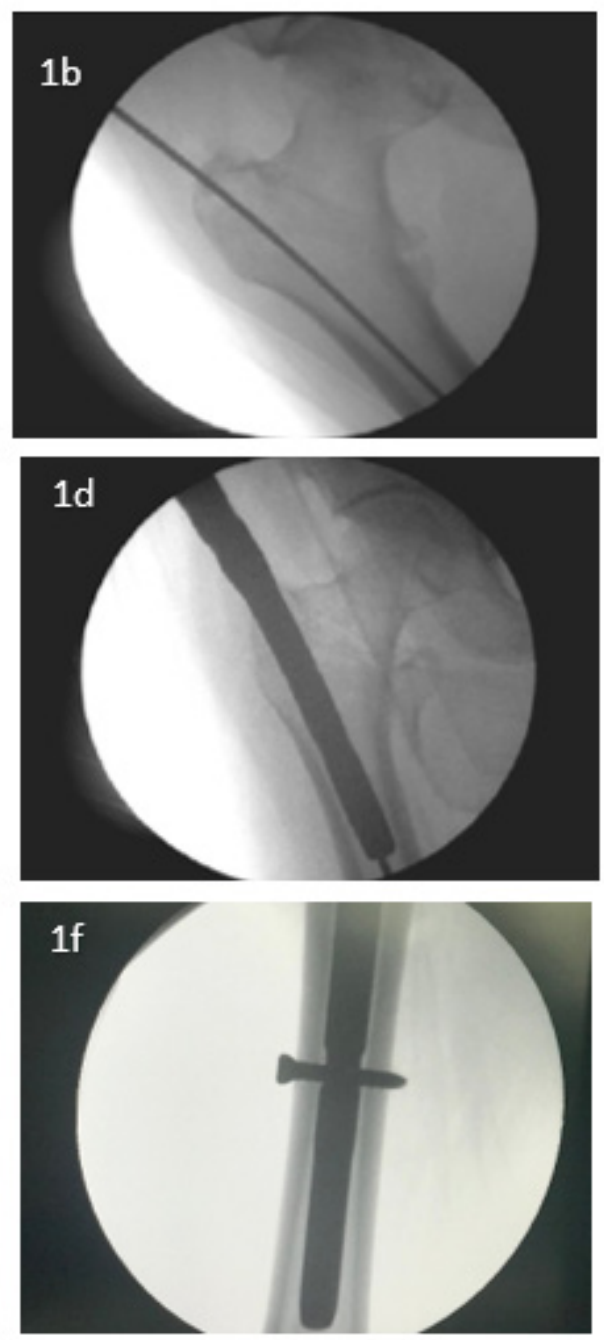

Figure 1: Surgical technique of PFN-A2 insertion - 1a) Nail entry point, 1b) Insertion of guide pin, 1c) Proximal reamer, 1d) Insertion of PFN-A2, 1e) Placement of helical blade and 1f) Placement of 4.9mm distal cortical screws 
All cases were given with IV antibiotics for 5 days followed by oral antibiotics for 5 days. The active and passive exercises were initiated within 48 hours of surgery. Tablet Risedronate $35 \mathrm{mg}$ once weekly for 12 weeks were given to improve the bone mineral density in patients with severe degree osteoporosis. All the patients were advised to completely weight bearing after 10-12 weeks of post-operative period. All patients were followed up at the end of 1, 2, 6, 12 and 18 months. At each follow up, the radiographs of upper femur and hip were taken to assess the fracture union and the complications. The functional results were calculated according to Harris hip score (Figure 2).

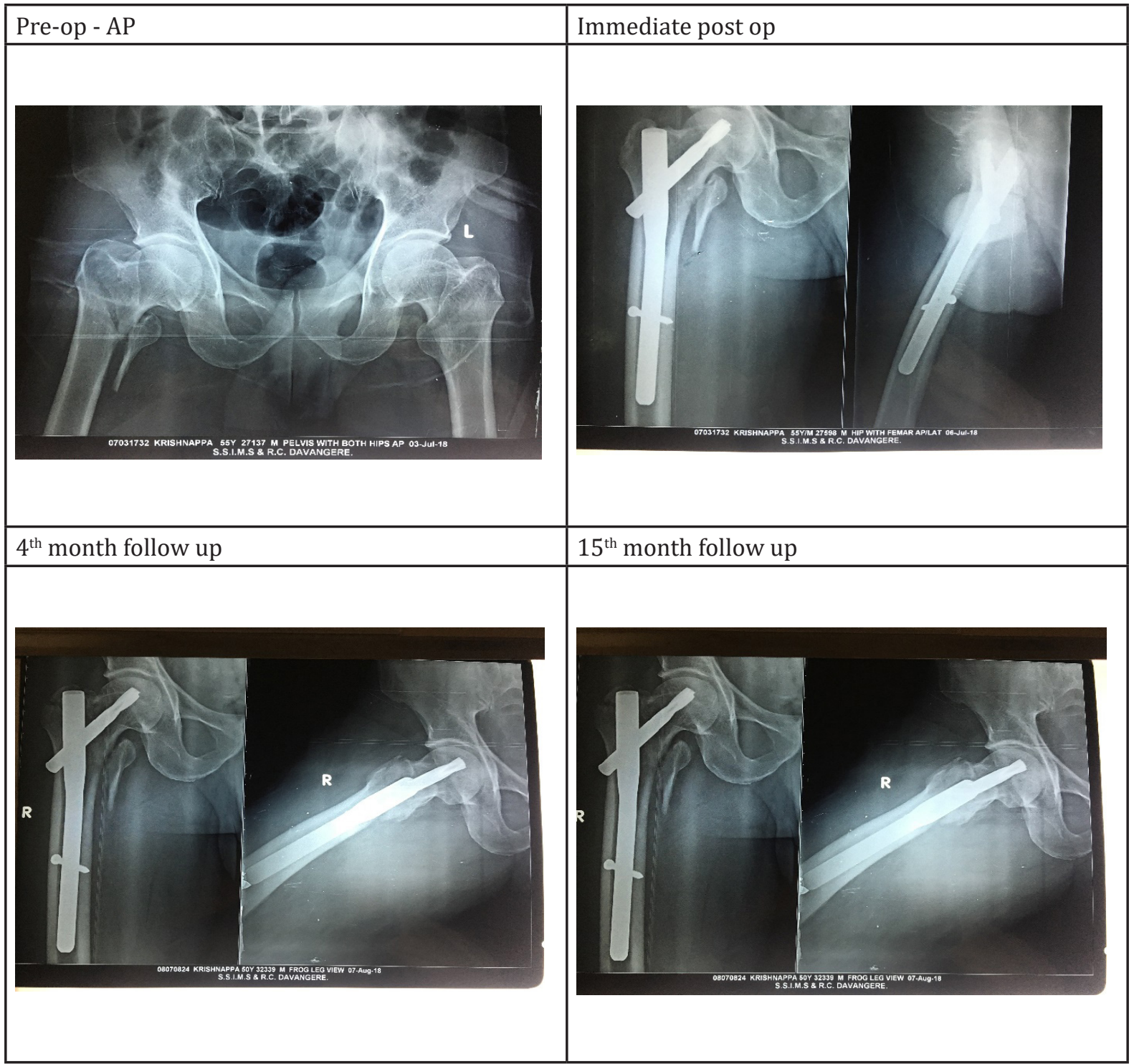

Figure 2: Radiographs of unstable intertrochanteric fracture fixed with PFN-A2.

\section{Results}

A total of 73 cases of unstable intertrochanteric fractures underwent surgical management with proximal femoral nail antirotation 2 (PFN-A2) as per our study protocol. The descriptive statistics were reported as mean (SD) for continuous variables, frequencies (percentage) for categorical variables. Data were statistically evaluated with IBM SPSS Statistics for Windows, Version 24.0, IBM Corp, Chicago, IL.

Among 73 cases, 47 cases (64.38\%) were males and 26 cases (35.61\%) were females. All the patients belong to age between
30 to 70 years of age. The maximum age limit in the study was 73 years and minimum age was 34 years. The mean $( \pm S D)$ age of the patients were $49.21 \pm 4.05$ years. A total of 37 cases $(50.68 \%)$ sustained injury due to road traffic accident, 17 cases $(23.28 \%)$ fall from height and 19 cases (26.02\%) due to trivial fall.

According to Boyd and Griffin classification, out of 73 cases of unstable intertrochanteric fractures, the pattern of fractures was type 3 in 28 cases (38.35\%) and type 4 in 45 cases (61.64\%). Among 73 cases, 59 cases (80.82\%) were operated with CRIF with PFN-A2 and 14 cases (19.17\%) underwent ORIF with PFN-A2. All cases were operated with an average of $4.09 \pm 0.91$ days ranging 
from 3.86 - 7.67 days of admission. The mean radiological union of unstable intertrochanteric fractures were $11.23 \pm 3.67$ weeks. A total of 2 cases $(2.73 \%)$ showed signs of established non-union after 9 months of post procedure with PFN-A2. These non-union cases were counselled for total hip arthroplasty. The complications associated in our study were pain 11 cases (15.06\%), non-union 2 cases $(2.73 \%)$, varus angulation 2 cases $(2.73 \%)$ and helical blade cut-out 1 case $(1.36 \%)$ (Figure 3$)$.

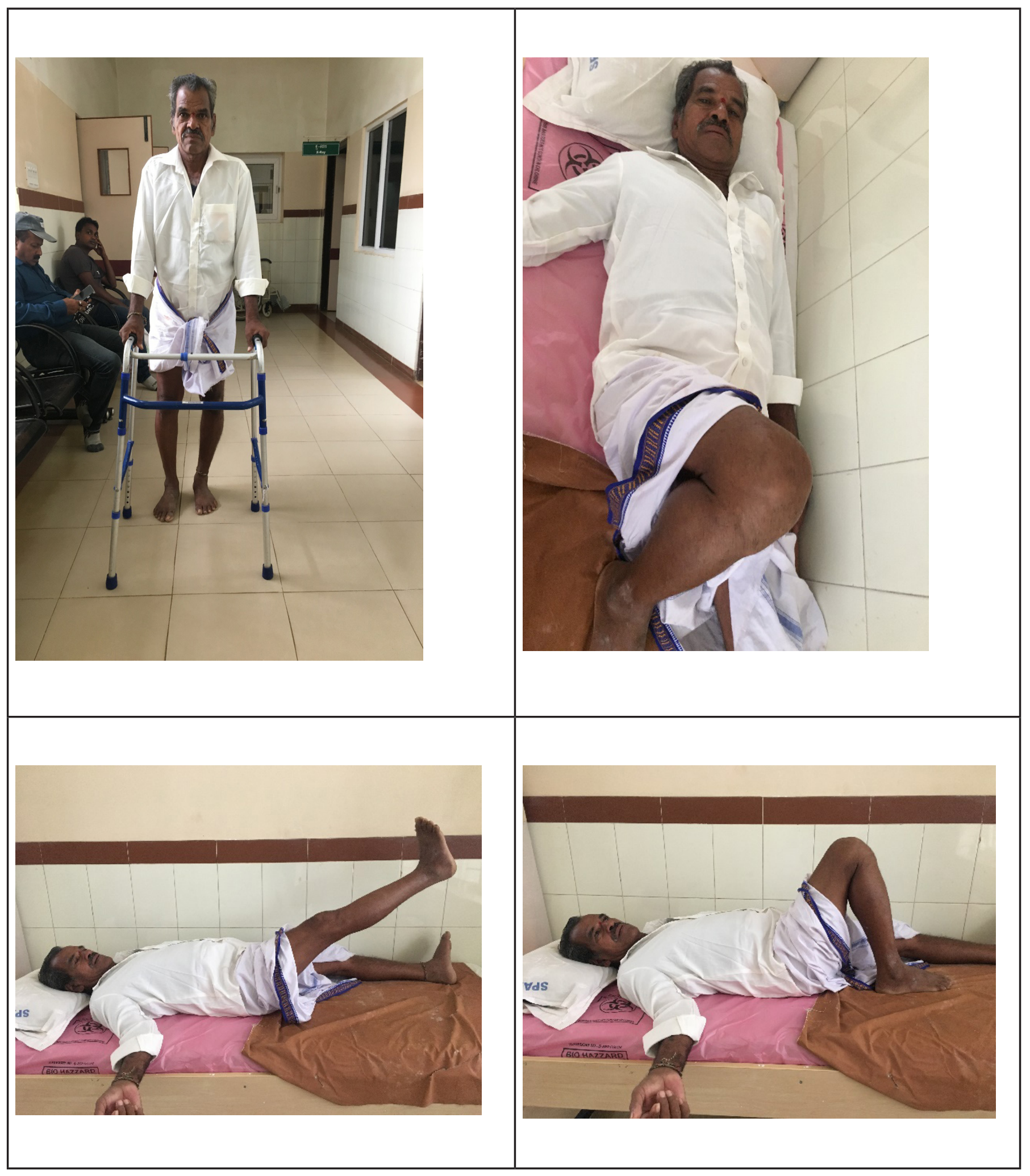

Figure 3: Clinical outcome in patient with of unstable intertrochanteric fracture fixed with PFN-A2. 
According to Harris Hip scores, out of 73 cases, the functional outcome was excellent in 41 cases (56.16\%), good in 15 cases (20.54\%), fair in 12 cases (16.43\%) and poor in 5 cases $(6.84 \%)$. The poor range of movements $(n=5)$ were due to non-union 2 cases, varus collapse 2 cases and helical blade cut-out 1 case.

\section{Discussion}

The management of unstable intertrochanteric fractures pose a significant challenge to the orthopaedic surgeon. These fractures represent a technical difficulty in reduction of fractures and implication of surgical implant may lead to failure of primary fixation of fracture. The medial and posteromedial fracture fragments were the most important elements in determining the severity of intertrochanteric fracture [10]. In this modern era, researchers stated that the important predictor for re-operation after the trochanteric fracture is the lateral femoral wall [11].

Cephalo-medullary femoral reconstruction nails with a trochanteric entry point are biomechanically stronger than extramedullary implants [12]. In unstable intertrochanteric fractures, the control of axial telescoping and rotational stability were essential. The recent implant for management of unstable intertrochanteric fracture was proximal femoral nail antirotation-2. PFN-A2 has advantages over PFNA in following ways: a) the proximal nail diameter was reduced from $17 \mathrm{~mm}$ to $16.5 \mathrm{~mm}$, b) the medio-lateral angle was reduced from 6 degree to 5 degree and c) a flat proximal lateral surface was adapted to avoid impingement of femoral lateral cortex $[13,14]$.

Bhatti et al. [15] concluded Proximal Femoral Nail was associated with reduced blood loss, shorter hospital stays, less morbidity compared with Dynamic Hip Screw. Klinger et al. [16] did a comparative study of unstable intertrochanteric fractures and concluded that Proximal Femoral Nail was associated with shorter operation time, shorter hospital stays, higher rate of patient with early full weight bearing, fewer complications compared with Dynamic Hip screw7.

Tornetta et al. [17] concluded that patients aged more than 65 years treated either with Gamma nail or a compression hip screw have no overall difference in functional outcomes. However, when patients with unstable fracture patterns were analyzed, those with an intramedullary nail had better walking ability at 12 months than those treated with compression hip screw.

Simmermacher et al. [18] concluded that PFNA currently is an optimal implant with regard to prevention of femoral head penetrations for the treatment of unstable trochanteric fractures. E. Soucanye de Landevoisin et al. [19] concluded that PFNA has additional benefits in patients with osteoporotic trochanteric fractures, both by preventing rotation and by ensuring cancellous bone compaction. This design may diminish the rate of complications associated with the cervical implant, provided the implantation procedure is scrupulously followed and fracture reduction is optimal.

Macheras et al. [20] concluded that PFNA II avoided lateral cortex impingement experienced with PFNA, providing fast and stable fixation of the unstable peritrochanteric fractures. Manoj et al. [21] concluded that both PFN and PFNA perform well, showing equally good functional outcomes following fixation of unstable trochanteric fractures. When compared to PFN, use of PFNA significantly reduces the duration of surgery, the amount of operative blood loss and fluoroscopic imaging.

Gururagavendra et al. [22] concluded that both the implants (PFN and PFN-A2) have comparable radiological and functional outcome for unstable intertrochantric fracture except for less surgical time and blood loss in PFN A2. Superiority of one implant over another could be arrived with still larger sample size, involving multi centre studies.

In our study, a new cephalo-medullary proximal femoral nail antirotation-2 (PFN-A2) for unstable intertrochanteric fractures were used. These nails prevent the rotation and collapse of the headneck fragment and smaller diameter of distal shaft of nail results in less stress concentration at the tip of the nail. The antirotation screw at the proximal aspect of nail increases the biomechanical stability of the fracture fixation. According to Harris Hip scores, out of 73 cases, the functional outcome was excellent in 41 cases (56.16\%), good in 15 cases (20.54\%), fair in 12 cases $(16.43 \%)$ and poor in 5 cases $(6.84 \%)$. PFN-A2 have the biological advantages in terms of restoration of abductor-lever-arm mechanism, decreased tensile strain on the implant and maintenance of controlled fracture impaction.

Limitations of the study were smaller sample size; limited duration of post-operative follow up to comment on biomechanical stability of the implant and no control group for comparison.

\section{Conclusion}

Unstable intertrochanteric fractures pose a great challenge for orthopaedic surgeons to manage surgically. Due to the evolution of load sharing devices, it is possible to manage the fractures with internal splinting. We conclude that the proximal femoral nail antirotation 2 (PFN-A2) was an ideal implant for unstable intertrochanteric fractures, leading to high rate of bone union restoring the lateral femoral wall, reducing the chances of implant failure and decreasing the post-operative morbidity by improving the functional quality of life.

\section{References}

1. Frederick MA, Terry SC, James HB. Campbell's operative orthopaedics in treatment of intertrochanteric fractures $\left(13^{\text {th }}\right.$ edn $)$, Vol. 3, p. 2828.

2. Sun-Jun H (2016) PENA-2 protrusion over the greater trochanter in the Asian population used in proximal femoral fractures. Ind J of Orthop 50(6): 641-646.

3. Franco L (2008) The treatment of stable and unstable proximal femoral fractures with a new trochanteric nail: result of a multicentre study with the Veronail. Strat Traum Recon 3(1): 15-22.

4. Adams CI, Robinson CM, Court-Brown CM (2001) Prospective randomized controlled trial of an intramedullary nail versus dynamic screw and plate of intertrochanteric fractures of the femur. J Orthop Trauma 15(6): 394-400. 
5. Gupta RK, Sangwan K, Kamboj P, Punia SS, Walecha P (2010) Unstable trochanteric fractures: the role of lateral wall reconstruction. Int Orthop 34(1): 125-129.

6. Li M, Wu L, Liu Y, Wang C (2014) Clinical evaluation of the asian proximal femur intramedullary nail antirotation system (PFNA-II) for treatment of intertrochanteric fractures. Journal of Orthopaedic Surgery and Research 9: 112.

7. Chang SM, Zhu XZ, Huang YG, Wang X, Zhang YQ, et al. (2010) Extramedullary DHS and intramedullary PFNA in unstable pertrochanteric fractures with lateral wall risky A0/0TA type 31A2.2 and A2.3: A clinical retrospective comparison. Orthop J China 18(22): 1868-1872.

8. Macheras GA, Koutsostathis SD, Galanakos SP, Kateros K, Papadakis SA (2013) Reply to letter to the editor: Does PFNA II avoid lateral cortex impingement for unstable peritrochanteric fractures? Clin Orthop Relat Res 471(4): 1395-1396.

9. Loo WL, Loh SYJ, Lee HC (2001) Review of Proximal Nail Antirotation (PFNA) and PFNA-2-Our local experience. Malaysian Orthopaedic Journal 5(2): 1-5.

10. Pajarinen J, Lindahl J, Michelsson O, Savolainen V, Hirvensalo E (2005) Pertrochanteric femoral fractures treated with a dynamic hip screw or a proximal femoral nail. A randomised study comparing post-operative rehabilitation. J Bone Joint Surg Br 87(1): 76-81.

11. Gotfried Y (2004) The lateral trochanteric wall: a key element in the reconstruction of unstable pertrochanteric hip fractures. Clin Orthop Relat Res 425: 82-86.

12. Linga SS, Aithala JP, Savur A (2017) Can proximal femoral nailing of unstable trochanteric fractures help to regain pre-injury functional status in Indian rural patients? A prospective study of functional outcome of unstable intertrochanteric fractures with proximal femoral nailing. Int J Res Orthop 3(4): 728-733.

13. Boblee J, Ram P, Vijayakumaran (2017) Functional outcome of proximal femoral nailing in intertrochanteric fractures of femur: A prospective study. Int J Orth Sci 3(2): 513-518.
14. Harshwardhan H, Jain S, Sharma M (2019) An outcome analysis of intertrochanteric fracture of femur managed with proximal femoral nail antirotation II. International Journal of Research in Orthopaedics 5(4): 699.

15. Bhatti A, Power D, Qureshi S, Khan I, Tan S (2004) A prospective trial of proximal femoral nail vs. dynamic hip screw for unstable intertrochanteric fractures of the femur. J Bone Joint Surg Br 86B(3): 377.

16. Klinger HM, Baums MH, Eckert M, Neugebaur R (2005) A comparative study of unstable per and intertrochanteric femoral fractures treated with Dynamic Hip Screws and trochanteric buttress plate vs. Proximal Femoral Nail Zentralbl Chir 130(4): 301-306.

17. Paul T, William R, Charles MCB, Margaret MQ, Michael M (2019) Rockwood and green fractures in adults ( $8^{\text {th }}$ edn), chapter 50, p. 2705.

18. Simmermacher RKJ (2008) The new proximal femoral nail antirotation (PFNA) in daily practice: Results of a multicentre clinical study Injury. Int J Care Injured 39(8): 932-939.

19. Soucanye de LE (2012) Proximal femoral nail antirotation (PFNATM) fixation of extra-capsular proximal femoral fractures in the elderly: Retrospective study in 102 patients. Orthopaedics \& Traumatology: Surgery \& Research 98: 288-295.

20. Macheras (2012) Does PFNA II avoid lateral cortex impingement for unstable peritrochanteric fractures? Clin Orthop Relat Res 470(11): 3067-3076.

21. Kashid MR, Gogia T, Prabhakara A, Jafri MA, Shaktawat DS, et al. (2016) Comparative study between proximal femoral nail and proximal femoral nail antirotation in management of unstable trochanteric fractures. Int J Res Orthop 2(4): 354-358.

22. Gururagavendra P, Sathish D, Jayakumar S, Devadoss A (2018) Randomised comparative study in management of unstable intertrochanteric fracture with PFN V/S PFN A2 - functional and radiological outcome. Int J Orthop Sci 4(4): 866-874.

For possible submissions Click below: 